\title{
FRED (A Framework for Reconstructing Epidemic Dynamics): an open-source software system for modeling infectious diseases and control strategies using census-based populations
}

John J Grefenstette ${ }^{1 *}$, Shawn T Brown², Roni Rosenfeld ${ }^{3}$, Jay DePasse ${ }^{2}$, Nathan TB Stone ${ }^{2}$, Phillip C Cooley ${ }^{4}$, William D Wheaton ${ }^{4}$, Alona Fyshe ${ }^{3}$ David D Galloway' ${ }^{1}$, Anuroop Sriram³ ${ }^{3}$ Hasan Guclu', Thomas Abraham ${ }^{3}$ and Donald S Burke ${ }^{1}$

\begin{abstract}
Background: Mathematical and computational models provide valuable tools that help public health planners to evaluate competing health interventions, especially for novel circumstances that cannot be examined through observational or controlled studies, such as pandemic influenza. The spread of diseases like influenza depends on the mixing patterns within the population, and these mixing patterns depend in part on local factors including the spatial distribution and age structure of the population, the distribution of size and composition of households, employment status and commuting patterns of adults, and the size and age structure of schools. Finally, public health planners must take into account the health behavior patterns of the population, patterns that often vary according to socioeconomic factors such as race, household income, and education levels.

Results: FRED (a Framework for Reconstructing Epidemic Dynamics) is a freely available open-source agent-based modeling system based closely on models used in previously published studies of pandemic influenza. This version of FRED uses open-access census-based synthetic populations that capture the demographic and geographic heterogeneities of the population, including realistic household, school, and workplace social networks. FRED epidemic models are currently available for every state and county in the United States, and for selected international locations.

Conclusions: State and county public health planners can use FRED to explore the effects of possible influenza epidemics in specific geographic regions of interest and to help evaluate the effect of interventions such as vaccination programs and school closure policies. FRED is available under a free open source license in order to contribute to the development of better modeling tools and to encourage open discussion of modeling tools being used to evaluate public health policies. We also welcome participation by other researchers in the further development of FRED.
\end{abstract}

Keywords: Pandemic influenza, Simulator, Agent-based model, Synthetic population, Influenza modeling

\footnotetext{
* Correspondence: gref@pitt.edu

${ }^{1}$ Graduate School of Public Health, University of Pittsburgh, Pittsburgh,

Pennsylvania, USA

Full list of author information is available at the end of the article
} 


\section{Background}

Mathematical and computational models provide valuable planning tools for public health challenges, especially for novel circumstances that cannot be examined through observational or controlled studies, such as pandemic influenza [1-12] or hypothetical bioterrorist attacks $[13,14]$. The development of models ideally involves a close working relationship between the modeling team and the decisionmaker using the model. Beyond the immediate outputs of a model itself, the modeling process itself can serve as a way of thinking through complex situations and clarifying assumptions [15]. While mathematical models have a long history of providing solid foundations for understanding disease dynamics [16], the tractability of analytic models may require neglecting heterogeneities in the population that may have important impacts on epidemic dynamics and on the effectiveness of possible interventions. For example, it has been suggested that attack rates for the 2009 H1N1 pandemic exhibited a high degree of spatiotemporal heterogeneities among different regions due to regional differences in socio-demographic factors [17]. In particular, the spread of infectious disease such as influenza depends on the mixing patterns within the population, and these patterns are in turn determined by numerous factors, including: population size and density $[18,19]$, the age structure of the population [20], the size and composition of households [21], school sizes and schedules [6,10,22-24], demographic and socioeconomic risk factors [25] including access to health care facilities $[9,11,26]$, employment patterns and policies [27], travel and commuting patterns $[12,28]$, and local behavioral practices including vaccine acceptance [26,29] and personal hygiene [30]. With these considerations in mind, public health officials may have particular interest in planning tools that take into account the specific characteristics of the local population of the region under their responsibility and that permit them to compare expected outcomes within their jurisdiction with expected outcomes in surrounding communities, or across an entire state.

This article describes FRED (a Framework for Reconstructing Epidemic Dynamics), a freely available opensource epidemic modeling system that uses census-based synthetic populations to capture the demographic and geographic heterogeneities of the population, including realistic household, school, and workplace social networks. FRED models are currently available for every state and county in the United States, and selected international locations. State and county public health planners can use FRED to explore the effects of possible influenza epidemics in their regions and to help evaluate the likely effect of interventions such as vaccination programs and school closure policies.

FRED represents a major software redesign and opensource release of epidemic models used in previously published studies by our team to evaluate potential responses to influenza pandemics, including vaccination policies [5,7-9], school closure $[6,10]$, the role of health care workers [11], and the effects of subway travel [12]. Building on these previous models, FRED was designed as a flexible framework for epidemic modeling, rather than a fixed model of a particular infectious disease. While originally designed to study influenza, FRED can be adapted to other infectious diseases, such as measles, by modifying configuration files characterizing the natural history of the disease. Other user-modifiable parameters include the initial immunological profile of the population, the availability and efficacy of vaccine and anti-viral drugs, and a flexible set of intervention policies regarding vaccine distribution, school closures and other non-pharmaceutical interventions. In addition, human behaviors in response to an epidemic can also be modeled in a variety of ways, from specifying simple probabilities that certain groups will get a vaccine or stay home from work or school when sick, to more sophisticated behavioral dynamics such as being influenced by concerns over a spreading epidemic.

\section{Implementation}

Key features of FRED include:

- Realistic synthetic populations based on the US Census Bureau's Public Use Microdata (PUMS) data and Census aggregated data. FRED is the first opensource epidemic model designed to use the latest synthetic US population developed by RTI [31].

- Highly modular, object-oriented software design to support rapid adaptation to a wide variety of infectious disease scenarios.

- Scalable and efficient simulation of large epidemics. FRED can be run on a variety of computer platforms from laptops to supercomputers, depending on the size of the population being simulated. Simulations of an influenza epidemic like the H1N1 pandemic in a population of 1 million people takes less than two minutes on a typical laptop computer.

- Multiple circulating strains can be simulated, making it suitable for the investigation of virus evolution, for example, antigenic drift or the evolution of resistant strains.

- Flexible ways to specify agent health behavior and decision rules. Agents in FRED may exhibit a number of health-related behaviors involving individual health decisions, such as staying home when sick, accepting a vaccine or taking an anti-viral drug. The FRED platform is designed to accommodate a range of models of health behavior and supports a variety of strategies to determine an agent's willingness to adopt a behavior [27]. 


\section{Synthetic population}

FRED explicitly represents every individual in a specific geographic region. For regions within the United States, FRED uses the 2005-2009 U.S. Synthetic Population Database (Version 2) from RTI International [31,32]. The synthetic population used an iterative fitting method [33] to generate an agent population from the US Census Bureau's Public Use Microdata files (PUMS) and aggregated data from the 2005-2009 American Community Survey (ACS) 5-year sample. The synthetic population contains geographically located synthetic households and household residents for the United States, as well as group quarters locations and residents (for college dorms, prisons, nursing homes, and military bases), schools and assignments of students to schools, workplaces and assignments of workers to workplaces. Each household, group quarters, school and workplace is mapped to a specific geographic location, reflecting the actual spatial distribution of the area and the distance travelled by individuals to work or to school $[34,35]$. Each agent has associated demographic and socioeconomic information (e.g., age, sex, race, household income) and locations for their activities (e.g., household, neighborhood, and possibly school or workplace). The number of elements for each category in the synthetic population is shown in Table 1.

The synthetic population closely matches the available census data for the United States with high spatial resolution. For example, the differences in the age of the head of household by county are shown in Figure 1. Overall the synthetic population differs from the ACS by less than $1 \%$ on this measure. Further detailed comparisons are provided in [31]. To illustrate the level of detail available for every county in the United States, Figure 2 shows various demographic distributions in the synthetic population for Allegheny County, PA.

The 2005-2009 U.S. Synthetic Population (Version 2) database is freely available, and synthetic populations are currently available from RTI for every state and county in the United States. Selected international locations, including Taiwan and Thailand, are available upon request from the authors. Users may apply FRED to other

\begin{tabular}{ll}
$\begin{array}{l}\text { Table } 1 \text { Elements in US synthetic population used in } \\
\text { FRED based on 2005-2009 American community } \\
\text { survey (ACS) }\end{array}$ \\
$\begin{array}{ll}\text { Individuals and } \\
\text { interaction groups }\end{array}$ & $\begin{array}{l}\text { Number in US } \\
\text { synthetic population }\end{array}$ \\
\hline Persons & $289,390,247$ \\
Households & $112,595,578$ \\
Workplaces & $10,696,738$ \\
Schools & 129,329 \\
\hline
\end{tabular}

populations not included in the synthetic population database by using the file formats specified in [32].

\section{Discrete-time simulation}

FRED performs a discrete-time simulation with time steps of one day, for any number of time steps. On each simulated day, each agent potentially interacts with the other agents who share the same activity locations. For example, school-age children in FRED interact with the same set of classmates at the school during each school day. If an infected agent interacts with a susceptible agent, there is a possibility of transmitting a disease from the infected agent to the susceptible agent. Each infection transmission event is recorded, making it possible to evaluate the effectiveness of several possible control measures and the impact on specific sub-populations. Agents may dynamically alter their daily activities, for example, by traveling or by deciding to stay home when sick.

The fixed simulation step of 1 day permits certain performance optimizations regarding scheduling the daily activities of agents and parallelizing the transmission of infection within places attended by disjoint sets of agents. The daily step size does not appear to be a severe limitation for running simulations that encompass several years for diseases with long latency periods, since the computation time per day depends primarily on the number of actively infectious individual on a given day. However, the daily step size may be a limitation for diseases with extremely short latency and infectious periods, or the simulation of short period (e.g. hourly) interventions.

\section{Agent model}

Each agent maintains a record of its demographic information (e.g., sex, race, date-of-birth, current age, employment or school status, family income), health information (e.g., current health status, list of infections, date of infection, level of symptoms, infectivity, susceptibility, immunity status, at-risk status), locations for social activity (household, neighborhood, and school or workplaces as appropriate), and health-related behaviors (e.g., probability of getting a vaccine or staying home when sick).

By default, the demographic features of agents in FRED remain constant during a given simulation run. However, some research questions may address epidemic dynamics over many years (e.g., how will a pandemic affect the population immunity over the next several years). To address these questions, FRED includes as an option some limited forms of dynamic agent demographics including aging, births and deaths. Age-specific maternity and mortality rate can be specified in external files. If dynamic demographics is enabled, then an agent's age may affect its activity pattern (e.g., school or work status) as well as the agent's health status (e.g., eligibility for a vaccine). Children that achieve school age are assigned to schools and adults 


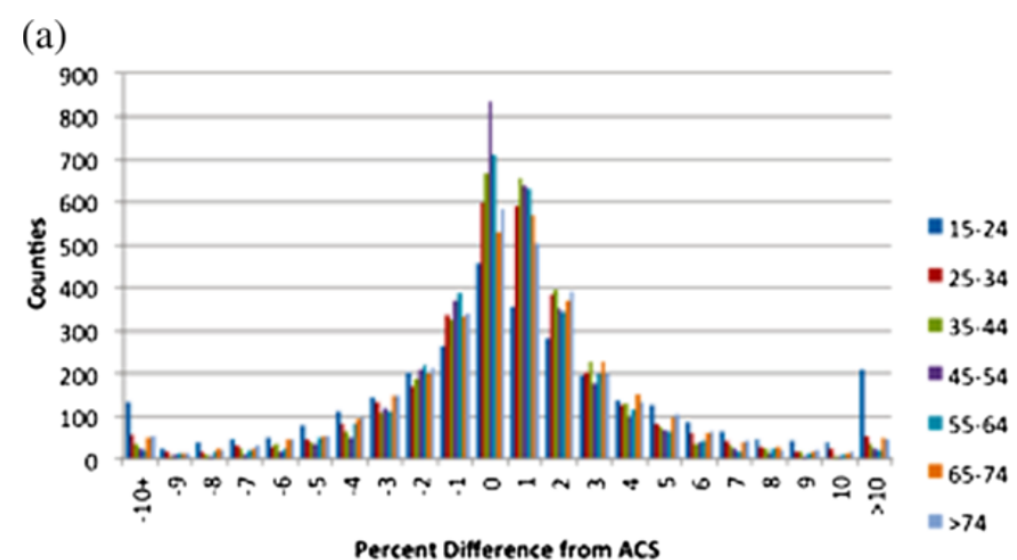

(b)

\begin{tabular}{|c|c|c|c|c|}
\hline & Min & Max & Mean & St Dev. \\
\hline$=15 \cdot 24$ & $-66.67^{*}$ & $200.00 * *$ & 0.08 & 9.05 \\
\hline$=25 \cdot 34$ & -40.48 & 47.83 & 0.06 & 4.49 \\
\hline$=35.44$ & -66.67 & 33.33 & 0.06 & 3.84 \\
\hline$=45.54$ & -22.08 & 25.00 & -0.04 & 2.88 \\
\hline$=55.64$ & -50.00 & 29.41 & 0.11 & 3.31 \\
\hline$=65 \cdot 74$ & -27.27 & 34.78 & 0.05 & 4.04 \\
\hline$>74$ & -60.00 & 33.33 & -0.08 & 4.43 \\
\hline
\end{tabular}

Figure 1 County level agreement between synthetic population and the American Community Survey (ACS). (a) Number of US counties with each percent difference in age of the head of household. (b) Mean and standard deviation over all counties of percentage differences by age of the head of household.

reaching working age are assigned to workplaces based on the attendance patterns in the agent's neighborhood. Newborns are assigned to the same household as their mother. If an agent dies, it is removed from the population. Further refinements to the dynamic demographics model, including household recombination and migration patterns, are under development.

Agents in FRED may exhibit a number of optional health-related behaviors including staying home from work or keeping a child home when sick, and accepting a vaccine for oneself or for a dependent child. At each time step, the action taken by an agent involves an interaction between the intention of the agent to perform the behavior and one or more external conditions such as the availability of a vaccine. In addition to describing an agent's intention as a simple probability as in previous models, FRED includes optional additional mechanisms for agent decision-making (Figure 3). As one example of using this flexible decision-making framework, FRED includes an implementation of the Health Belief Model, in which health behavior decisions are based on several specific constructs including perceived susceptibility, severity, benefits, and barriers [36]. These constructs are implemented as FRED perceptions and are combined into an agent-specific decision rule as described in [37]. The behavioral features of FRED are under active development, but some initial results showing the importance of behavioral heterogeneities within the population are available [27], described in the Results section below.

\section{Place model}

FRED assumes that all disease-specific interactions among agents occur in a specific place, and each type of place represents a distinct environment for the spread of infection. The FRED framework includes a generic Place class that can be instantiated into subclasses as needed by the model developer for a particular study. The default types of places in FRED include households, neighborhoods, schools, and workplaces, but these are not intended as an exhaustive list of places that may be important sites of infection. Neighborhoods are defined on a grid with 
(a)

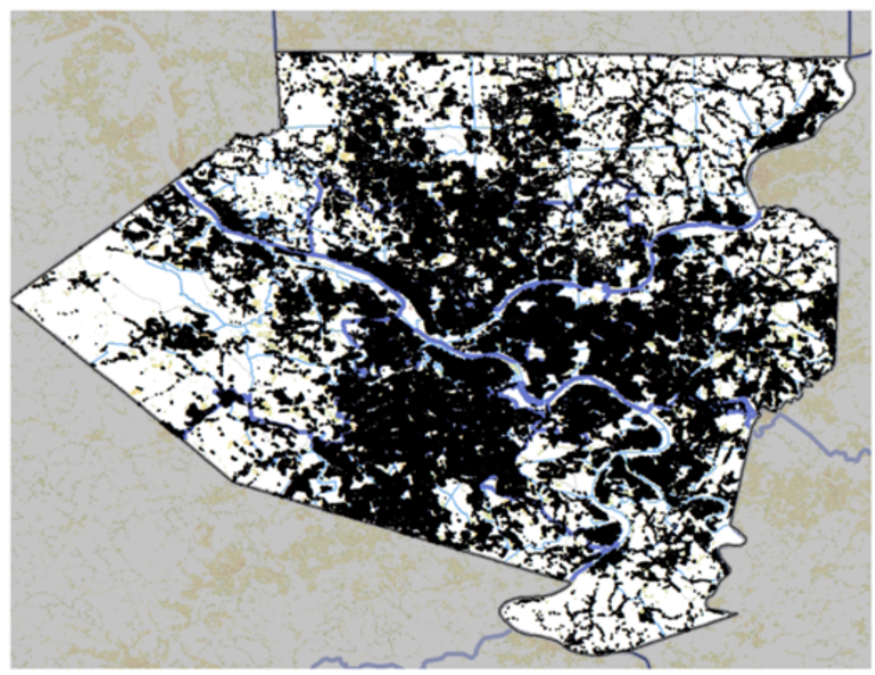

(b)

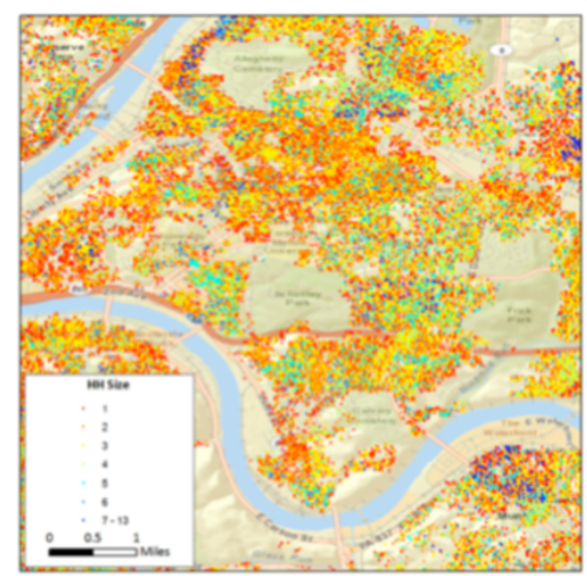

Household Size

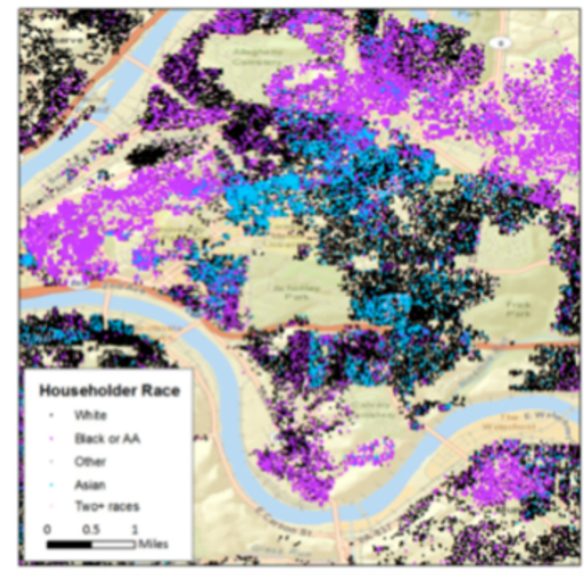

Race

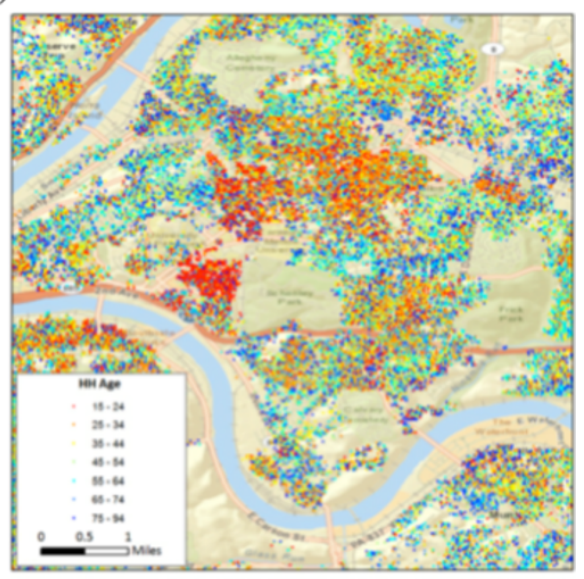

Age

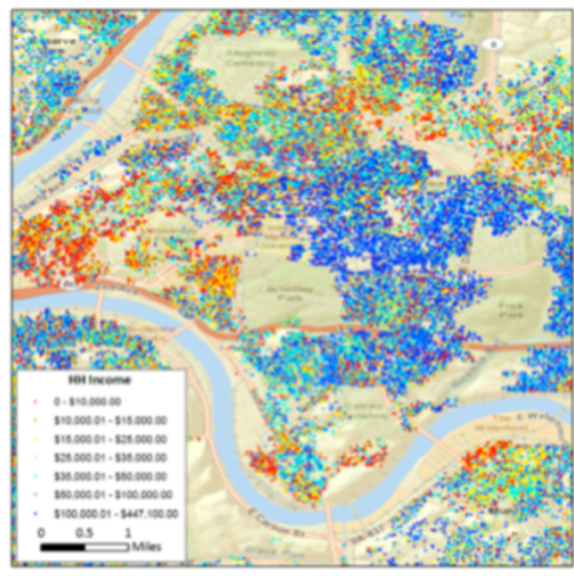

Income

Figure 2 Demographic features in Allegheny County synthetic population. (a) Overall population density in Allegheny County. (b) Spatial distribution by household size, age of householder, race of householder, and household income. 


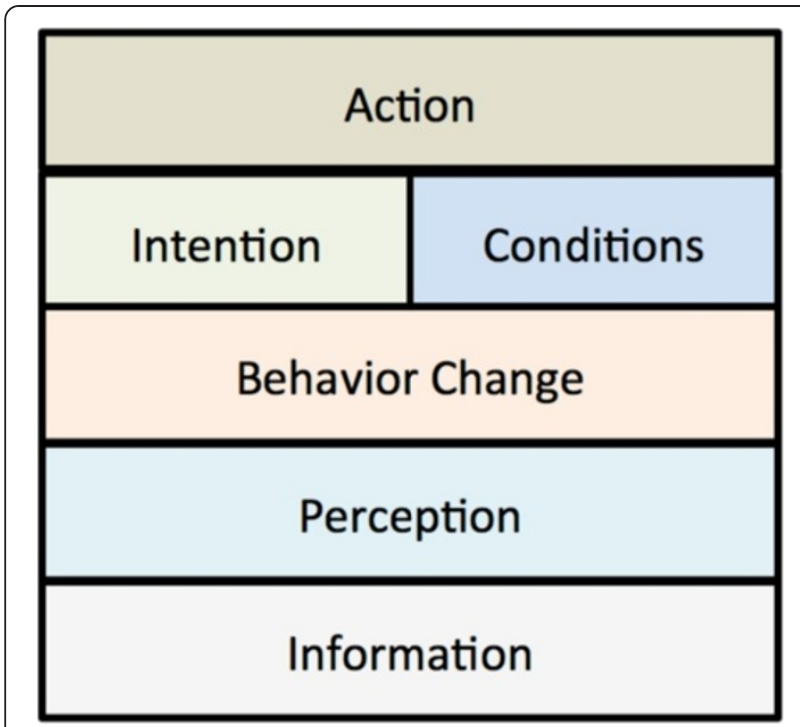

Figure 3 Mechanisms for agent-specific health decision-making in FRED. Agents can query the information layer to assess, for example, the current incidence, resulting in a perception ("how susceptible am I to the disease?"). Perceptions can be used by a behavior change model that determines whether to change the agent's intention to perform the health-related behavior. These features permits the FRED developer to investigate a wide variety of alternative health behavior change models, including the Health Belief Model [36,37].

$1 \mathrm{~km}$ square cells. Agents tend to spend their neighborhood activities within their home neighborhoods, defined as the cell in which the agent's household is located, but agents may also visit other neighborhood during a given day, according to a modified gravity model. FRED also provides optional classrooms (small mixing groups within a given school) and offices (small mixing groups within a given workplace). Nonworkplace contacts in workplaces (e.g., customers) are not currently supported, but will be included in a future version of the framework.

Depending on an assigned activity profile (for example, student, worker, retiree, etc.), each agent maintains a default daily schedule of places that the agent visits on a regular basis (e.g., the agent's household, neighborhood, school or workplace). Agent may visit different places depending on the day of the week, the time of year, or ad hoc travel plans. As in previous models [5-12], schools are closed on weekends and during scheduled summer holidays. Similarly, most workers do not visit their workplaces on weekends. However, some workers are designated as weekend workers, and they continue to visit workplaces on weekends. To reflect weekend schedules of schools and workplaces, the number of neighborhood contacts is increased by $50 \%$ on weekends [12].

\section{Disease model}

The FRED framework supports the circulation in the population of one or more infectious diseases. Each disease is specified by a set of natural history, contact and transmission parameters (Table 2). The default distribution includes disease parameters for pandemic influenza derived from previously published influenza models [12]. All parameters are in Table 2 are user-modifiable based on the specific disease being modeled.

For a given agent, an infection is assumed to follow a user-specified temporal pattern, with the agent typically assuming the standard S-E-I-R pattern of susceptible, exposed (infected but not infectious to others), infectious, and recovered (or removed) states. It is also possible to specify the rate at which agents lose immunity after recovery. The distribution of the number of days spent in each state is specified by user-settable parameters. Thus disease patterns such as S-E-I-R-S or S-E-I-S can be modeled. FRED supports multiple strains circulating in the same population. The intensity and temporal trajectory of cross-immunity among strains, as well as its dependence on the genetic or antigenic distance between the strains, can be specified by the user. A detailed, equation-based intra-agent infection model is also available, so that the susceptibility, infectivity and symptoms of an agent can depend on the details of an agent's exposure and treatment history. Other options, such as extending the Intrahost class to support more complex disease models, are described in the system documentation.

If an agent is infectious on a given day, then any place the agent visits during that day is considered a potentially infectious location. The place-specific transmission model is described in Figure 4. Susceptible agents can only become infected at a potentially infectious location, so interactions among agents at non-infectious locations need not be simulated.

The rate of effective contacts (that is, the number of transmission events per infectious individual) in a given place depends on two place-specific parameters: the expected number of contacts per infectious person per day, and the probability that a contact transmits an infection. The expected number of contacts per day depends on the place type but not on the place size. For example, students in a small school are assumed to have the same number of contacts per day as students in a large school. The place-specific transmission probabilities depend on the ages of the agents involved. This permits the model to reflect, for example, that an infectious child in school is more likely to infect another student than to infect a teacher, even if the infectious child contacts both. By default these parameters are set as in previous models [5-12]. If an infectious agent has multiple contacts with a given susceptible agent (for example, as members of the same household or same 
Table 2 User-modifiable disease-specific parameters

\begin{tabular}{|c|c|c|}
\hline Parameter type & Parameter & Definition \\
\hline \multirow[t]{6}{*}{ Natural history parameters } & Days latent & Discrete cdf for number of days between becoming exposed and becoming infectious \\
\hline & Symptomatic rate & The probability of an infected person becoming symptomatic \\
\hline & Days asymptomatic & Discrete cdf for number of days the agent is infectious but asymptomatic \\
\hline & Days symptomatic & Discrete cdf for number of days the agent is infectious and symptomatic \\
\hline & Immunity loss rate & Rate at which a person loses immunity after recovering from infection \\
\hline & Mortality rate & The probability of an infected person dying \\
\hline \multirow[t]{5}{*}{ Contact parameters } & Probability of staying home & The baseline probability that an agent stays home if the agent experiences a symptomatic infection. \\
\hline & Household contact rates & $\begin{array}{l}\text { The expected number of potentially infective daily contacts between an infectious agent and a } \\
\text { susceptible agent in a household. All contact rates are positive real numbers. }\end{array}$ \\
\hline & Neighborhood contact rates & $\begin{array}{l}\text { The expected number of potentially infective daily contacts between an infectious } \\
\text { agent and a susceptible agent in a neighborhood }\end{array}$ \\
\hline & School contact rates & The expected number of potentially infective daily contacts between an infectious agent and a susceptible agent in a school \\
\hline & Workplace contact rates & $\begin{array}{l}\text { The expected number of potentially infective daily contacts between an infectious agent and a } \\
\text { susceptible agent in a workplace. }\end{array}$ \\
\hline \multirow[t]{6}{*}{ Transmission parameters } & Transmissibility & The transmissibility of disease relative to an arbitrary baseline set by calibration \\
\hline & Asymptomatic infectivity & Multiplier for how infective an asymptomatic infected agent is, relative to an symptomatic agent \\
\hline & Household transmission probability & $\begin{array}{l}\text { A table of probabilities that a potentially infective contact between an infectious agent and a } \\
\text { symptomatic agent in the same household actually results in an infection, given the age of the potential infector/infectee pair }\end{array}$ \\
\hline & Neighborhood transmission probability & $\begin{array}{l}\text { A table of probabilities that a potentially infective contact between an infectious agent and a symptomatic agent occurring } \\
\text { in a neighborhood actually results in an infection, given the age of the potential infector/infectee pair }\end{array}$ \\
\hline & School transmission probability & $\begin{array}{l}\text { A table of probabilities that a potentially infective contact between an infectious agent and a symptomatic } \\
\text { agent occurring in a school } \\
\text { actually results in an infection, given the age of the potential infector/infectee pair }\end{array}$ \\
\hline & Workplace transmission probability & $\begin{array}{l}\text { A table of probabilities that a potentially infective contact between an infectious agent } \\
\text { and a symptomatic agent occurring in a workplace actually results in an infection, } \\
\text { given the age of the potential infector/infectee pair }\end{array}$ \\
\hline
\end{tabular}




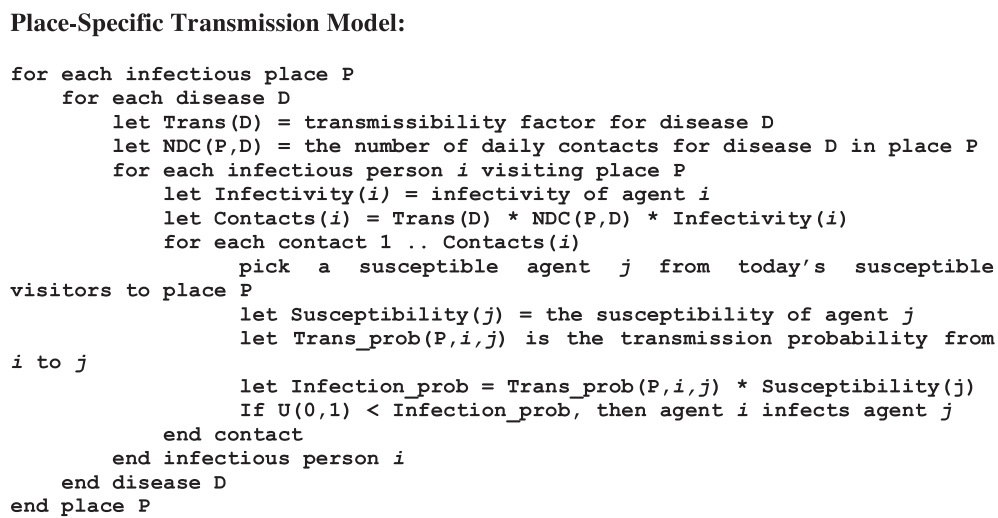

Figure 4 Pseudo-code for the place-specific transmission model in FRED.

classroom), each such contact is considered as an independent opportunity to transmit the infection.

Epidemics in FRED are initiated by seeding the population with one or more infections. These infections may either be assigned to members of the population selected randomly, or restriction to sub-groups defined by an age distribution or a selected geographical area. To account for the impact of an epidemic in populations external to modeled population, the user may specify a time-varying schedule of cases that causes FRED to seed new infections into the study area throughout the course of the epidemic.

\section{Performance and scalability}

Computational efficiency is an important concern when modeling the potential interactions of millions of individuals. As in other large-scale epidemic models, FRED obtains much of its efficiency by focusing its transmission kernel only on the active set of infectious individuals and their interactions with susceptible individuals. FRED adopts a few additional significant optimizations:

1. Since every disease transmission occurs within a given place in FRED, we only apply the placespecific transmission model (Figure 4) to potentially infectious locations, that is, locations that are visited by at least one infectious individual during the current simulation day.

2. Once all potentially infectious locations are identified, the transmission model can be applied to all such locations of a given type in parallel. Simulating transmission in parallel in all infectious locations of a given type (e.g. all schools) ensures that no agent occurs in two such locations at the same time (e.g. all children attend at most one school). This avoids potential timing issues that may arise if all infectious locations were simulated in parallel.

3. FRED uses a shared memory multi-threaded parallel model implemented with OpenMP, which allows simulations on a quad-core computer with hyperthreading (i.e., many current laptops) to run approximately four times faster than with a purely serial implementation.

FRED requires between 750 and 1000 megabytes of memory per million simulated individuals. The exact amount of memory required depends on the demographic and geographic characteristics of the synthetic population, as well as the severity of the simulated epidemic. Simulations of an influenza spread like the H1N1 pandemic in a population of 1 million people takes less than two minutes on a typical dual-core laptop computer but the runtime will vary depending on the number of individuals infected during the epidemic and depending on which optional features are selected. On the supercomputer Blacklight at the Pittsburgh Supercomputer Center (an SGI Altix UV shared-memory architecture with up to $16 \mathrm{~TB}$ of shared memory), a simulated pandemic over the entire U.S. population requires approximately $200 \mathrm{~GB}$ of memory and takes approximately 4 hours using 16 threads.

The synthetic populations for individual states and the District of Columbia range in size from approximately 600,000 individuals for Washington, DC to over 30,000,000 individuals in California. To demonstrate how FRED scales with population size, we performed FRED simulations of the 50 states and the District of Columbia using default influenza parameters and no intervention. Figure 5 shows that runtime scales linearly over two orders of magnitude in population size. Given FRED's memory requirement of about $750 \mathrm{MB}$ to $1 \mathrm{~GB}$ per million agents, 30 million agents (e.g., California) can be simulated on a workstation with about $24 \mathrm{~GB}$ of memory.

\section{Implementation details}

FRED is written in the $\mathrm{C}++$ programming language and is released under the BSD 3-Clause Open Source License 


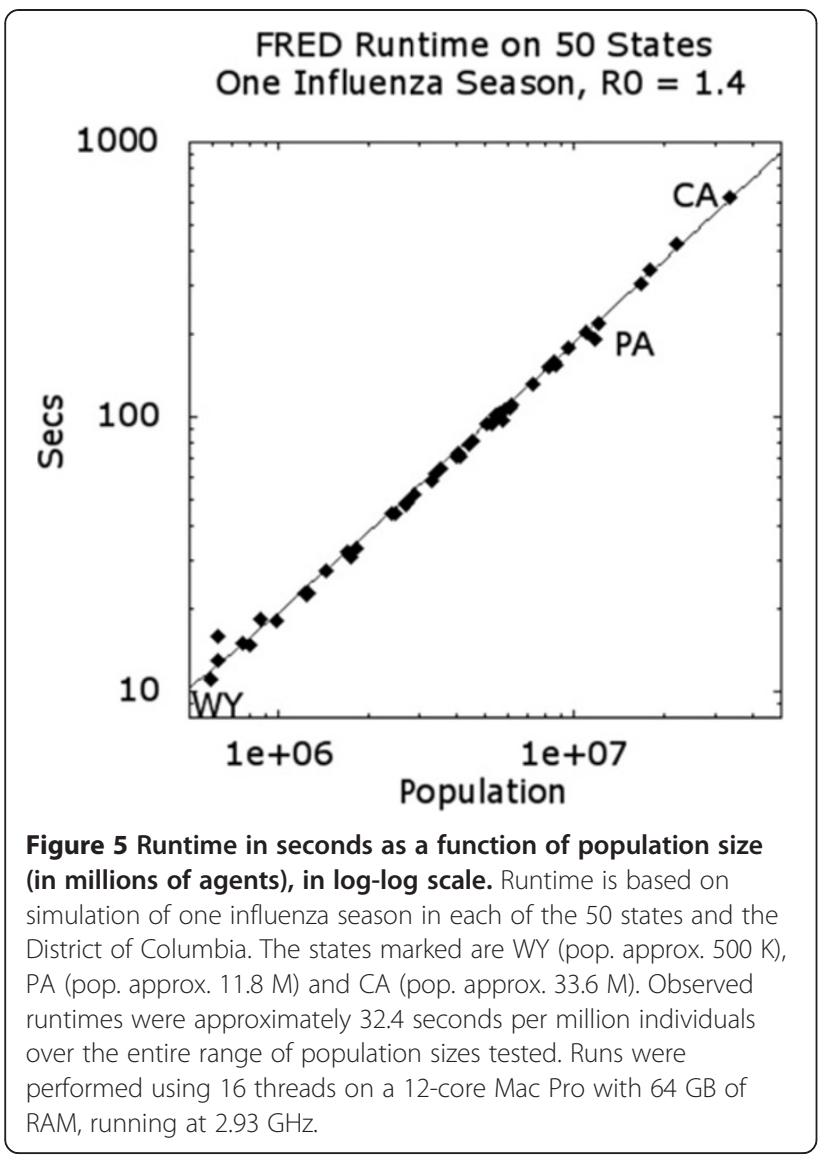

(http://opensource.org/licenses/BSD-3-Clause). The current distribution, available in Additional file 1, includes the FRED source code, documentation with installation instructions, tutorials on using the software, and detailed descriptions of all the configuration parameters. The documentation also describes the programming model and includes source level documentation and other details for developers who may be interested in extending the FRED framework for their own use.

The primary output file contains one line for each simulation day of the run, displaying a large selection of output variables. Optional additional files record the infection history of each infected individual, including the identity of the infecting individual, the place of infection and other details. The documentation describes the formats of the output files and how to modify the source code to include other variables if desired. FRED includes plotting scripts that display time series for any selected output variables.

FRED is distributed with sample synthetic populations, including Allegheny County (Pittsburgh), PA. Synthetic populations for other regions are available online as part of the 2005-2009 U.S. Synthetic Population (Version 2) [31]. Version 2.2.1 of FRED was used to produce the results in this paper. The latest version of the FRED distribution is available online at fred.publichealth.pitt.edu.

\section{Results}

Effects of schools closures during an influenza pandemic To compare the current version of FRED with our previously published models, we reproduced studies from [6] that evaluated the potential effectiveness of alternative school closure policies during a pandemic influenza in Allegheny County, Pennsylvania. In particular, we considered policies that closed a given school when a number of sick students were observed at that school. The FRED software distribution includes a parameterization for pandemic influenza as used in previous models [5-12]. As described in detail in [12], place-specific contact parameters were calibrated using a 30-70 rule [3] in which $30 \%$ of all transmissions are assumed to occur in the household, 33\% in the general community and $37 \%$ in schools and workplaces, and the fraction of transmissions that occur in schools is twice of those that occur in workplaces. The system was calibrated to reproduce a pandemic with a 50\% Attack Rate (AR) in a completely susceptible population while satisfying the 30-70 rule. The baseline model assumed that $50 \%$ of sick individuals withdraw to their home and do not interact with anyone outside of the household, consistent with previous models [5-12],

In this study, a given school was closed when the number of sick children at that school reached a trigger value (fixed at 10 for this example). We compared the effects of varying the duration of the school closure once it was initiated at each school. Specifically, the duration of school closure was varied from 2 to 8 weeks, and once schools reopened, they did not close again. Figure 6 shows the daily incidence (number of new infections) for each scenario. Note that the curves for all school closure scenarios

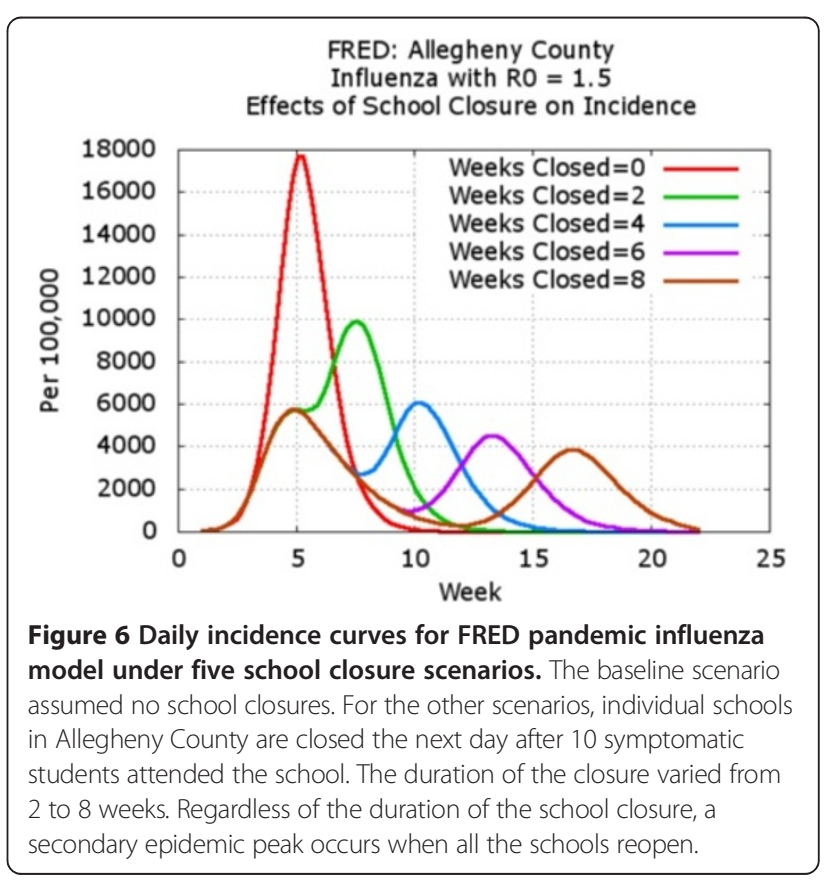


are essentially identical through the first 5 weeks of the epidemic, at which time the scenarios with shorter school closure durations begin to reopen schools. It can be observed that for all school closure policies, the epidemic temporarily abated when schools closed, but peaked again once the schools had reopened. The resulting attack rate (i.e. the total percentage of people that became infected through the course of the epidemic) is shown in Figure 7. While the daily incidence temporarily declined during the period corresponding to school closures, the final attack rate was similar for all scenarios, reflecting the resurgence of the epidemic once schools reopen. These results were consistent with our previously published model [6], although some details differed due to changes in the synthetic population model.

\section{Simulation of epidemics on all US counties}

To illustrate the effect of regional differences on epidemic dynamics, we downloaded the synthetic population files for every county in the US and performed a FRED simulation of a baseline pandemic influenza epidemic in each county using the default parameters as discussed above. By using the same transmission parameters for all counties, we can see some of the effects of local heterogeneities in the population mixing patterns across the US. As expected, we observed a range of resulting attack rates for the 3142 counties tested (Figure 8). The mean attack rate (using 20 independent runs per county) for all counties was $48.4 \%$ (std. dev. 6.7). Counties with extremely small populations exhibited the extreme values for attack rate, with a minimum attack rate of $6.6 \%$ (for Catron County, NM; pop. 3202 ) and a maximum attack rate of $76.8 \%$ (Wade Hampton Census Area, Alaska; pop. 7203), perhaps

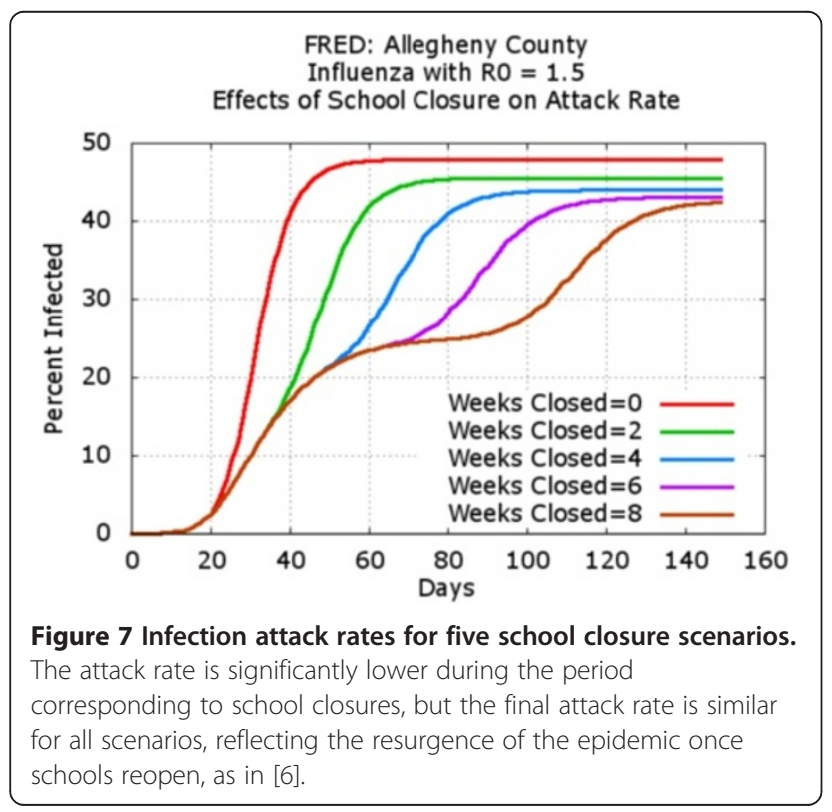

showing the particularly strong effects of contact patterns in small populations. However, even among counties with populations over 500,000 a wide range of attack rates were observed, from a minimum of $37.9 \%$ for San Francisco County, CA (pop. 682,007) to a maximum attack rate of $68.0 \%$ for Hidalgo County, TX (pop. 701,751). These results suggest that health officials may want to consider the likely effect of interventions in the context of the local population structure.

To encourage further exploration of these simulation results, we have made FRED simulations of US counties available at fred.publichealth.pitt.edu. A user can browse thousands of previously run simulations (Figure 9), or run a new FRED influenza simulation with other combinations of epidemic parameters and control measures. In addition to incidence, prevalence and attack rate curves, the user can visualize results via maps and movies showing the epidemic dynamics for the given location via the GAIA webservice [Additional file 2]. Web users can also download FRED output data and perform their own analysis.

\section{Health-related behaviors and policies}

A recent article [27] showed the possibilities for using FRED to investigate the impact of employment-related health policies on the health of both the workers directly involved and on the general population during an influenza pandemic. In this study, agents in FRED were assigned a probability of staying home based on the available of paid sick days (PSD) at their workplace. Based on data from the US Bureau of Labor Statistics [38], the model assumed that employees had access to PSD depending on the size of their workplace and that $72 \%$ of employees who had access to PSD and $52 \%$ of

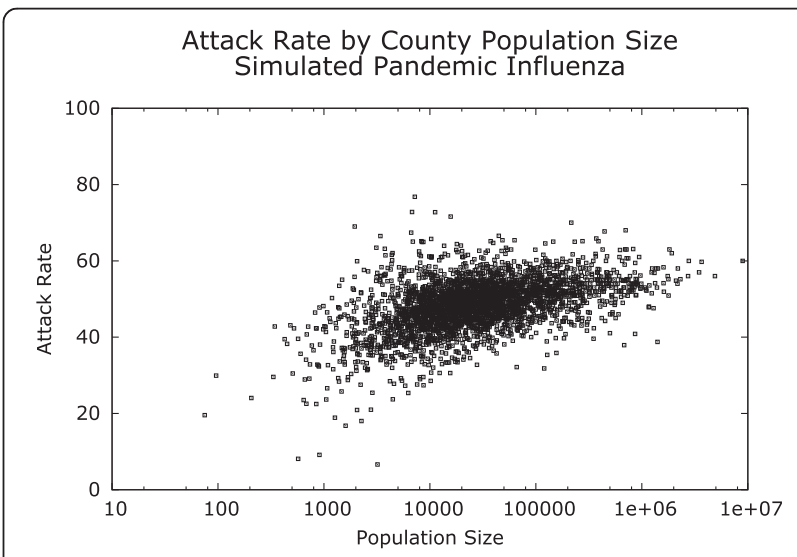

Figure $\mathbf{8}$ Infection attack rate for $\mathbf{3 1 4 2}$ counties in the United States, using FRED's baseline pandemic influenza transmission parameters. The plot shows the mean attack rate for each county over 20 stochastic simulations. The attack rate displays significant heterogeneity across US counties. 


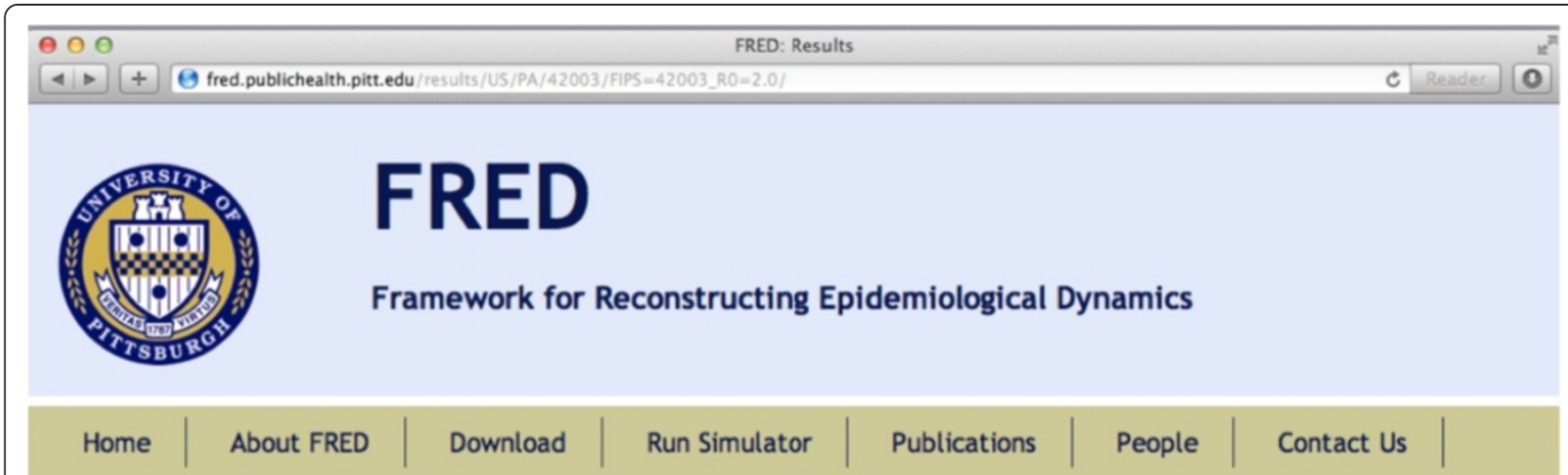

\section{FRED Results for: FIPS=42003_R0=2.0}

\section{Parameters:}

\section{\#\# Location}

county = Allegheny County, PA

fips $=42003$

popsize $=1164880$

\#\# Epidemic Parameters

$\mathrm{RO}=2.0$

initial_cases $=10$

days $=\overline{9} 0$

\#\# Control Parameters

Immunization $=0.0$

Download data:

- incidence.csv

- symptomatic_incidence.csv

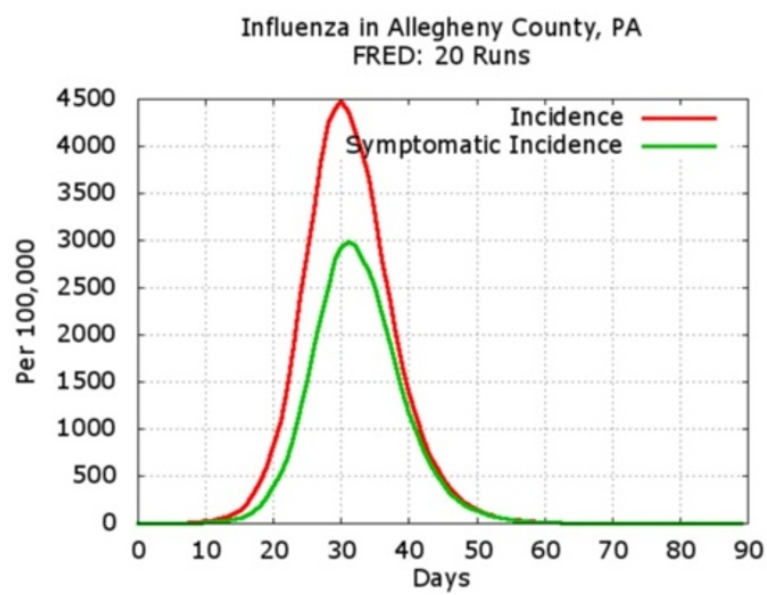

Influenza in Allegheny County, PA Day (25)

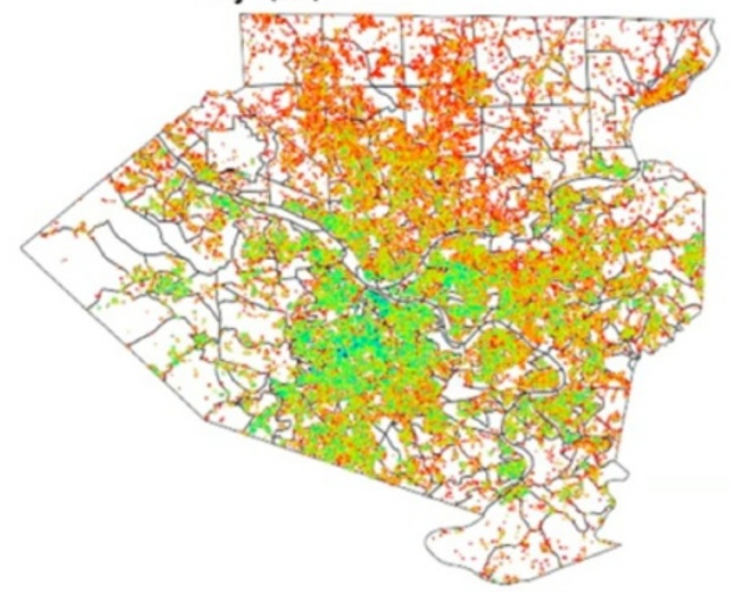

Influenza in Allegheny County, PA FRED: 20 Runs

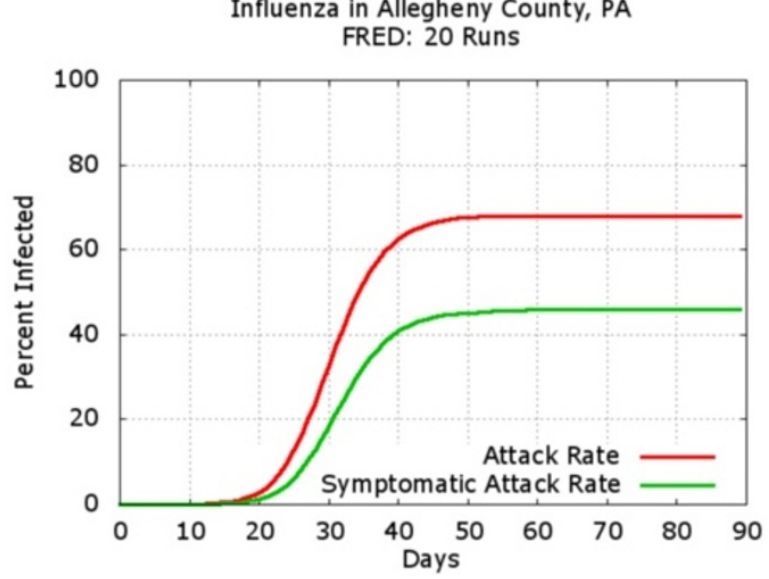

Figure 9 Website showing results of FRED simulations. Results available for an influenza simulation in Allegheny County, PA at fred. publichealth.pitt.edu. Similar results are available for every county in the US. Users can also specify epidemic parameters and control parameters for additional simulations, and can download data files to perform additional analyses. 
those without PSD stayed home when ill with influenza. The average amount of days off from work was also set on the basis of an observational study [39]. Simulations for Allegheny County, PA, showed that a large proportion (72\%) of the workplace attack rate was due to exposure to other employees engaging in presenteeism, defined as going to work when symptomatic. It was also shown that providing universal PSD to all employees reduced workplace infections by $5.86 \%$, with larger reductions in infection occurring in small workplaces (with 2-49 employees) than in large workplaces (with 500 employees or more). Finally, the results showed that providing one or two additional "flu days" (allowing employees with influenza to stay home) reduced workplace infections by about $25 \%$ and $39 \%$, respectively. This study illustrates the importance of considering heterogeneities in the health-related behavior of individuals and in the workplace environments when considering the population-level impact of alternative policy interventions.

\section{Discussion}

FRED simulations suggest that differences in population structure, spatial distribution, and other local factors can produce significant differences in the spread on infection disease among the counties in the U.S. We believe that these examples illustrate some of the advantages inherent in agent-based models that use data-driven population models, compared to simpler compartmental models that neglect heterogeneities present in real populations.

Several other epidemic simulation programs have been made available in open source form, including FluTE [40], EpiFire [41], GEM [42] and GSAM [43]. We believe that FRED offers an attractive combination of features that make it a valuable additional to the research community, including:

- A free, open source license

- Use of realistic, open-access, census-based synthetic populations

- Scalability from laptops to supercomputers

- Highly efficient simulation with populations up to hundreds of millions of agents

- An interface with the GAIA visualization system (http://gaia.psc.edu)

- Support for multiple circulating strains within the population, making it suitable for the investigation of virus evolution, for example, antigenic drift or the evolution of resistant strains.

FRED is under active development, and several additional features are planned for future versions that will extend its value as a tool for public health planning, including:
- Additional health-related behavior models, supporting the further study of how human behavior impacts potential public health control measures, and how health-related behaviors change over time.

- Long-term dynamic demographics of the agent population, such as migration patterns and household changes such as marriages and divorce, enabling the study of long-term health behavior patterns as well as chronic diseases such as tuberculosis.

- Vector-borne diseases, including dengue and malaria.

- A more flexible simulation time step.

- Automated workflows for advanced probabilistic sensitivity analysis [44].

In addition to the FRED web site, we have created an auxiliary tool called FRED Navigator [45] that allows the user to explore the effects of changing simulation parameters by interactively browsing through a database of simulation results. FRED Navigator is aimed at making FRED a practical tool for the public health user and a teaching tool for students in public health. We invite interested parties to contribute to the development of FRED and to extend its use as a tool for public health decision-making, research and education.

As with any model, users should take appropriate cautions to understand the limitations of FRED. Limitations of FRED include stochastic effects that limit the accuracy of the synthetic population especially in regions with very small populations [32], possible artifacts due to the selected time step resolution (one day), simplifying assumptions about travel patterns (gravity model), and the fact that estimates of contact rates and transmission probabilities are necessarily imperfect, even if based on estimates from the literature. Models created with FRED are stochastic, so results may vary from run to run, and some events, especially early in an epidemic, may depend on random choices such as the identity of the initial cases. Understanding the scope of variability in complex models such as FRED is an active area of research [44-46]. FRED supports the process of uncertainty analysis by providing workflow management scripts for setting up parameter sweeps and performing local sensitivity analysis.

\section{Conclusions}

FRED (a Framework for Reconstructing Epidemic Dynamics) is a freely available open source epidemic modeling platform based on several previously developed influenza models. FRED simulates epidemics within a census-based synthetic population that reflects the specific population characteristics of a given region, including spatial distribution, race, age, and household income, along with realistic household, school, and workplace contact networks. FRED allows the flexible specifications of disease characteristics, 
intervention strategies, and a variety of health-related behaviors. These features make FRED a valuable tool for public health planners to explore possible epidemic scenarios in a specific jurisdiction and to evaluate the possible effects of interventions such as vaccination programs and school closure policies. We hope that the availability of FRED will contribute to the further development of modeling tools for public health decision support. We particularly welcome suggestions from the user community on ways to make FRED a more useful tool for planning public health responses to epidemics.

\section{Availability and requirements \\ Project name: FRED}

Project home page: http://fred.publichealth.pitt.edu Operating system(s): OS X, Linux, Windows (under Cygwin)

Programming language: $\mathrm{C}++$, Python and Perl. Other requirements: Optional plotting features require Gnuplot.

License: BSD Open Source License

Any restrictions to use by non-academics: None.

\section{Additional files}

Additional file 1: FRED Distribution Version 2.2.1. This file contains the FRED distribution, including source files, installation instructions, required input files, and documentation. Unpack the file and see the file FRED/README.txt for further installation instructions.

Additional file 2: Quicktime movie. GAIA Visualization of FRED simulation of Allegheny County. This file contains a Quicktime movie showing the prevalence of influenza in Allegheny County resulting from a FRED simulation calibrated to an R0 of 2.0. The movie was produced by the GAIA visualization tool (http://gaia.psc.edu). The FRED distribution contains scripts that automate the generation of GAIA visualization from FRED simulations.

\section{Abbreviations}

ACS: American community survey; FRED: A framework for reconstructing epidemic dynamics; PUMS: US census bureau's public use microdata.

\section{Competing interests}

The authors declare that they have no competing interests.

\section{Authors' contributions}

JJG, STB and RR designed the software architecture, based on original code developed by PCC and STB. WDW and PCC provided the interface to the synthetic population. JDP, NTBS, AF, DDG, AS, and TA contributed to the development of the software. HG contributed to the analysis of FRED components. DSB provided design goals and coordination for the project. JJG drafted the manuscript. All authors read and approved the final manuscript.

\section{Acknowledgements}

This work was supported by the National Institute of General Medical Sciences under MIDAS grant U54GM088491 and by the Vaccine Modeling Initiative, funded by the Bill and Melinda Gates Foundation. The funders had no role in study design, data collection and analysis, decision to publish, or preparation of the manuscript. The authors wish to thank the reviewers for their thoughtful comments that helped to improve the presentation of the article.

\section{Author details}

${ }^{1}$ Graduate School of Public Health, University of Pittsburgh, Pittsburgh, Pennsylvania, USA. ${ }^{2}$ Pittsburgh Supercomputing Center, Carnegie Mellon University, Pittsburgh, Pennsylvania, USA. ${ }^{3}$ School of Computer Science, Carnegie Mellon University, Pittsburgh, Pennsylvania, USA. ${ }^{4}$ RTI International, Research Triangle Park, Durham, North Carolina, USA.

Received: 11 June 2013 Accepted: 25 September 2013

Published: 8 October 2013

\section{References}

1. Eubank S, Guclu H, Kumar VS, Marathe MV, Srinivasan A, Toroczkai Z, Wang $\mathrm{N}$ : Modelling disease outbreaks in realistic urban social networks. Nature 2004, 429(6988):180-184

2. Ferguson NM, Cummings DA, Cauchemez S, Fraser C, Riley S, Meeyai A, lamsirithaworn S, Burke DS: Strategies for containing an emerging influenza pandemic in Southeast Asia. Nature 2005, 437(7056):209-214.

3. Ferguson NM, Cummings DA, Fraser C, Cajka JC, Cooley PC, Burke DS: Strategies for mitigating an influenza pandemic. Nature 2006, 442(7101):448-452.

4. Halloran ME, Ferguson NM, Eubank S, Longini IM Jr, Cummings DA, Lewis B, Xu S, Fraser C, Vullikanti A, Germann TC, Wagener D, Beckman R, Kadau K, Barrett C, Macken CA, Burke DS, Cooley P: Modeling targeted layered containment of an influenza pandemic in the United States. Proc Nat Acad Sci U S A 2008, 105(12):4639-4644.

5. Lee BY, Brown ST, Cooley P, Grefenstette JJ, Zimmerman RK, Zimmer SM, Potter MA, Rosenfeld R, Wheaton WD, Wiringa AE, Bacon KM, Burke DS: Vaccination deep into a pandemic wave potential mechanisms for a "third wave" and the impact of vaccination. Am J Prev Med 2010, 39(5):e21-e29.

6. Lee BY, Brown ST, Cooley P, Potter MA, Wheaton WD, Voorhees RE, Stebbins S, Grefenstette JJ, Zimmer SM, Zimmerman RK, Assi TM, Bailey RR, Wagener DK, Burke DS: Simulating school closure strategies to mitigate an influenza epidemic. J Public Health Manag Pract 2010, 16(3):252-261.

7. Lee BY, Brown ST, Cooley PC, Zimmerman RK, Wheaton WD, Zimmer SM, Grefenstette JJ, Assi TM, Furphy TJ, Wagener DK, Burke DS: A computer simulation of employee vaccination to mitigate an influenza epidemic. Am J Prev Med 2010, 38(3):247-257.

8. Lee BY, Brown ST, Korch GW, Cooley PC, Zimmerman RK, Wheaton WD, Zimmer SM, Grefenstette JJ, Bailey RR, Assi TM, Burke DS: A computer simulation of vaccine prioritization, allocation, and rationing during the 2009 H1N1 influenza pandemic. Vaccine 2010, 28(31):4875-4879.

9. Lee BY, Brown ST, Bailey RR, Zimmerman RK, Potter MA, McGlone SM, Cooley PC, Grefenstette JJ, Zimmer SM, Wheaton WD, Quinn SC, Voorhees RE, Burke DS: The benefits to all of ensuring equal and timely access to influenza vaccines in poor communities. Health affairs 2011, 30(6):1141-1150.

10. Brown ST, Tai JH, Bailey RR, Cooley PC, Wheaton WD, Potter MA, Voorhees RE, LeJeune M, Grefenstette JJ, Burke DS, McGlone SM, Lee BY: Would school closure for the $2009 \mathrm{H} 1 \mathrm{~N} 1$ influenza epidemic have been worth the cost?: a computational simulation of Pennsylvania. BMC Public Health 2011, 11:353.

11. Cooley P, Lee BY, Brown S, Cajka J, Chasteen B, Ganapathi L, Stark JH, Wheaton WD, Wagener DK, Burke DS: Protecting health care workers: a pandemic simulation based on Allegheny county. Influenza Other Respi Viruses 2010, 4(2):61-72

12. Cooley P, Brown S, Cajka J, Chasteen B, Ganapathi L, Grefenstette J, Hollingsworth CR, Lee BY, Levine B, Wheaton WD, Wagener DK: The role of subway travel in an influenza epidemic: a New York City simulation. J Urban Health 2011, 88(5):982-995.

13. Burke DS, Epstein JM, Cummings DA, Parker JI, Cline KC, Singa RM, Chakravarty S: Individual-based computational modeling of smallpox epidemic control strategies. Acad Emerg Med 2006, 13(11):1142-1149.

14. Longini IM Jr, Halloran ME, Nizam A, Yang Y, Xu S, Burke DS, Cummings DA, Epstein JM: Containing a large bioterrorist smallpox attack: a computer simulation approach. Int J Infect Dis 2007, 11(2):98-108.

15. Burke DS, Grefenstette JJ: Towards an integrated meta-model of public health dynamics for preparedness decision support. J Public Health Manag Pract 2013, 19(Suppl 2):S12-5.

16. Keeling M, Rohani P: Modeling Infectious Diseases in Humans and Animals. Princeton: Princeton University Press; 2007.

17. Merler S, Ajelli M, Pugliese A, Ferguson NM: Determinants of the spatiotemporal dynamics of the $2009 \mathrm{H} 1 \mathrm{~N} 1$ pandemic in Europe: implications for real-time modelling. PLoS Comput Biol 2011, 7(9):e1002205. 
18. Suryaprasad A, Redd JT, Hancock K, Branch A, Steward-Clark E, Katz JM, Fry AM, Cheek JE: Severe acute respiratory infections caused by 2009 pandemic influenza A (H1N1) among American Indians-southwestern United States, May 1-July 21, 2009. Influenza Other Respi Viruses 2013 : 10.1111/irv.12123. [Epub ahead of print]

19. Yu H, Feng L, Viboud CG, Shay DK, Jiang Y, Zhou H, Zhou M, Xu Z, Hu N, Yang W, Nie S: Regional variation in mortality impact of the 2009 A (H1N1) influenza pandemic in China. Influenza Other Respi Viruses 2013: 10.1111/irv.12121. [Epub ahead of print]

20. Stark JH, Sharma R, Ostroff S, Cummings DA, Ermentrout B, Stebbins S, Burke DS, Wisniewski SR: Local spatial and temporal processes of influenza in Pennsylvania, USA: 2003-2009. PloS one 2012, 7(3):e34245.

21. Cauchemez S, Donnelly CA, Reed C, Ghani AC, Fraser C, Kent CK, Finelli L, Ferguson NM: Household transmission of 2009 pandemic influenza A (H1N1) virus in the United States. N Engl J Med 2009, 361(27):2619-2627.

22. Cauchemez S, Valleron AJ, Boelle PY, Flahault A, Ferguson NM: Estimating the impact of school closure on influenza transmission from Sentinel data. Nature 2008, 452(7188):750-754

23. Chao DL, Halloran ME, Longini IM Jr: School opening dates predict pandemic influenza $\mathrm{A}(\mathrm{H} 1 \mathrm{~N} 1)$ outbreaks in the United States. J Infect Dis 2010, 202(6):877-880.

24. Araz OM, Damien P, Paltiel DA, Burke S, van de Geijn B, Galvani A, Meyers $L A$ : Simulating school closure policies for cost effective pandemic decision making. BMC public health 2012, 12:449.

25. Zimmerman RK, Lauderdale DS, Tan SM, Wagener DK: Prevalence of highrisk indications for influenza vaccine varies by age, race, and income. Vaccine 2010, 28(39):6470-6477.

26. Davila-Payan C, Swann J, Wortley PM: System factors to explain H1N1 state vaccination rates for adults in US emergency response to pandemic. Vaccine 2013. S0264-410X(13)00684-1. doi: 10.1016/j. vaccine.2013.05.069. [Epub ahead of print]

27. Kumar S, Grefenstette JJ, Galloway D, Albert SM, Burke DS: Policies to reduce influenza in the workplace: impact assessments using an agentbased model. Am J Public Health 2013, 103(8):1406-1411.

28. Stark JH, Cummings DA, Ermentrout B, Ostroff S, Sharma R, Stebbins S, Burke DS, Wisniewski SR: Local variations in spatial synchrony of influenza epidemics. PloS one 2012, 7(8):e43528.

29. Shim E, Grefenstette JJ, Albert SM, Cakouros BE, Burke DS: A game dynamic model for vaccine skeptics and vaccine believers: measles as an example. J Theor Biol 2012, 295:194-203.

30. Stebbins S, Cummings DA, Stark JH, Vukotich C, Mitruka K, Thompson W, Rinaldo C, Roth L, Wagner M, Wisniewski SR, Dato V, Eng H, Burke DS: Reduction in the incidence of influenza $A$ but not influenza $B$ associated with use of hand sanitizer and cough hygiene in schools: a randomized controlled trial. Pediatr Infect Dis J 2011, 30(11):921-926.

31. Wheaton WD: 2005-2009 U.S. Synthetic Population Ver. 2. RTI International; 2012. http://www.epimodels.org/midas/Rpubsyntdata1.do.

32. Wheaton WD: U.S. Synthetic Population Database 2005-2009: Quick Start Guide. RTI International; 2012. http://portaldev.rti.org/10_Midas_Docs/ SynthPop/2005-2009_synth_pop_ver2_quickstart.pdf.

33. Beckman RJ, Baggerly KA, McKay MD: Creating synthetic baseline populations. Transp Res A Policy Pract 1996, 30(6):415-429.

34. Wheaton WD, Cajka JC, Chasteen BM, Wagener DK, Cooley PC, Ganapathi L, Roberts DJ, Allpress JL: Synthesized population databases: A US geospatial database for agent-based models, Research Triangle Park, NC. RTI Press; 2009. http://www.rti.org/pubs/mr-0010-0905-wheaton.pdf.

35. Cajka JC, Cooley PC, Wheaton WD: Attribute Assignment to a Synthetic Population in Support of Agent-Based Disease Modeling. Methods Rep 2010, 19(1009):1-14. RTI.

36. Durham DP, Casman EA, Albert SM: Deriving behavior model parameters from survey data: self-protective behavior adoption during the 20092010 influenza A(H1N1) pandemic. Risk Anal 2012, 32(12):2020-2031.

37. Durham DP, Casman EA: Incorporating individual health-protective decisions into disease transmission models: a mathematical framework. J R Soc Interface 2012, 9(68):562-570.

38. U.S. Bureau: of Labor Statistics: Employee Benefits in the United States March 2010.

39. Palmer LA, Rousculp MD, Johnston SS, Mahadevia PJ, Nichol KL: Effect of influenza-like illness and other wintertime respiratory illnesses on worker productivity: the child and household influenza-illness and employee function (CHIEF) study. Vaccine 2010, 28(31):5049-5056.
40. Chao DL, Halloran ME, Obenchain VJ, Longini IM Jr: FluTE, a publicly available stochastic influenza epidemic simulation model. PLoS Comput Biol 2010, 6(1):e1000656.

41. Hladish T, Melamud E, Barrera LA, Galvani A, Meyers LA: EpiFire: an open source $\mathrm{C}++$ library and application for contact network epidemiology. BMC Bioinformatics 2012, 13:76.

42. Epstein JM, Goedecke DM, Yu F, Morris RJ, Wagener DK, Bobashev GV: Controlling pandemic flu: the value of international air travel restrictions. PloS one 2007, 2(5):e401.

43. Parker J, Epstein JM: A distributed platform for global-scale agent-based models of disease transmission. ACM Trans Model Comput Simul 2011, 22(1):25.

44. Nsoesie EO, Beckman RJ, Marathe MV: Sensitivity analysis of an individual-based model for simulation of influenza epidemics. PloS one 2012, 7(10):e45414.

45. Paparian J, Brown S, Burke D, Grefenstette J: FRED Navigator: An interactive system for visualizing results from large-scale epidemic simulations. In Proceedings of the 8th International Conference on e-Science (e-Science 2012): 8-12 Oct 2012. Chicago: IEEE; 2012:1-5.

doi:10.1186/1471-2458-13-940

Cite this article as: Grefenstette et al:: FRED (A Framework for

Reconstructing Epidemic Dynamics): an open-source software system for modeling infectious diseases and control strategies using censusbased populations. BMC Public Health 2013 13:940.

\section{Submit your next manuscript to BioMed Central and take full advantage of:}

- Convenient online submission

- Thorough peer review

- No space constraints or color figure charges

- Immediate publication on acceptance

- Inclusion in PubMed, CAS, Scopus and Google Scholar

- Research which is freely available for redistribution

Submit your manuscript at www.biomedcentral.com/submit
C) Biomed Central 